\title{
Interferon Signaling Process
}

National Cancer Institute

\section{Source}

National Cancer Institute. Interferon Signaling Process. NCI Thesaurus. Code C40586.

Any process which promotes expression of the interferon proteins, their subsequent secretion, binding to cognate receptors or intracellular signaling, and promotes antigen presentation by $T$ lymphocytes, interference with viral replication, and activation of NK cells and macrophages. This process is involved in modulating functions of the immune system. 